OHSTPY-HEP-T-03-001

February 2003

\title{
A Natural Framework for Bi-large Neutrino Mixing
}

\author{
S. Raby \\ Department of Physics, The Ohio State University, \\ 174 W. 18th Ave., Columbus, Ohio 43210
}

\begin{abstract}
In this letter we present a "natural" framework for obtaining bi-large neutrino mixing incorporating the Frampton-Glashow-Yanagida neutrino mass matrix ansatz. We show that an $S U(2) \times U(1)$ family symmetry can provide the desired FGY neutrino mass ansatz in the MSSM. We also show how to obtain an approximate FGY ansatz in an $S O(10)$ SUSY GUT. In this context, the same $S U(2) \times U(1)$ family symmetry also generates the hierarchy of fermion masses as well as ameliorating SUSY flavor problems.
\end{abstract}


In a recent paper [1] [FGY] a simple ansatz for neutrino masses has been presented which has several significant virtues. It is an elegant framework for so-called bi-large neutrino mixing, i.e. giving maximal $\nu_{\mu}-\nu_{\tau}$ mixing for atmospheric neutrinos and large mixing angle Mikheyev-Smirnov-Wolfenstein mixing for solar neutrinos. ${ }^{1}$ In addition, the sign of the cosmological baryon asymmetry is related to $\mathrm{CP}$ violation possibly observable in low energy neutrino oscillation experiments. ${ }^{2}$ In this letter we present a simple $S U(2) \times$ $U(1)$ symmetry within the context of supersymmetry which makes the FGY ansatz "natural". We then extend this simple realization to an $S O(10)$ grand unified theory [GUT] which also fits charged fermion masses and mixing angles. In the latter example, the possibility of additional $\mathrm{CP}$ violating angles destroys the leptogenesis/neutrino $\mathrm{CP}$ violation connection, but still provides a natural framework for bi-large neutrino mixing.

Consider the three lepton doublets $l_{i}, i=1,2,3$. Under an $S U(2)$ family symmetry, two of the lepton doublets transform as a doublet given by $L_{a}=\left(\begin{array}{l}l_{1} \\ l_{2}\end{array}\right)$, while $l_{3}$ is a singlet. In addition, we require two flavor (anti)-doublets $\phi^{a}$ and $\tilde{\phi}^{a}, 4$ flavor singlets $N_{1}, N_{2}, S_{1}, S_{2}$ and the standard model Higgs doublet $H$.

The superpotential is given by

$$
W=\frac{H}{M}\left(L_{a} \phi^{a} N_{1}+L_{a} \tilde{\phi}^{a} N_{2}\right)+b^{\prime} H l_{3} N_{2}+\frac{1}{2}\left(S_{1} N_{1}^{2}+S_{2} N_{2}^{2}\right)
$$

where $M$ is some large mass scale. Of course for the charged leptons we need the standard Yukawa couplings to the Higgs doublets. We assume that the charged lepton Yukawa matrix is diagonal, so that the neutrinos $\nu_{i}$ are in the lepton flavor basis. The $U(1)$ charge assignments for the fields $\left\{L_{a}, l_{3}, N_{1}, N_{2}, \phi^{a}, \tilde{\phi}^{a}, S_{1}, S_{2}\right\}$ are as follows $\{1,-y, x, y,-(x+1),-(y+1),-2 x,-2 y\}$ with $x \neq y$ and $x+y+1 \neq 0$.

We assume $\phi, \tilde{\phi}$ get vevs $\langle\phi\rangle=\left(\begin{array}{c}\phi^{1} \\ \phi^{2}\end{array}\right)$ and $\langle\tilde{\phi}\rangle=\left(\begin{array}{c}0 \\ \tilde{\phi}^{2}\end{array}\right)$. This does not require fine tuning since any arbitrary vevs can be rotated into this direction using an $S U(2) \times U(1)$ flavor rotation. The vevs $\left\langle S_{i}\right\rangle=M_{i}, i=1,2$ are also needed to give the states $N_{i}$ a large see-saw mass. These vevs can be obtained via suitable terms added to the superpotential. We then obtain the $3 \times 2$ mixing matrix $D^{t r}$ defined by

$$
\left(\begin{array}{lll}
\nu_{1} & \nu_{2} & \nu_{3}
\end{array}\right) D^{t r}\left(\begin{array}{l}
N_{1} \\
N_{2}
\end{array}\right) \equiv\left(\begin{array}{lll}
\nu_{1} & \nu_{2} & \nu_{3}
\end{array}\right)\left(\begin{array}{cc}
a & 0 \\
a^{\prime} & b \\
0 & b^{\prime}
\end{array}\right)\left(\begin{array}{l}
N_{1} \\
N_{2}
\end{array}\right)
$$

where

$$
a=\frac{v}{\sqrt{2}} \frac{\phi^{1}}{M}, \quad a^{\prime}=\frac{v}{\sqrt{2}} \frac{\phi^{2}}{M}, \quad b=\frac{v}{\sqrt{2}} \frac{\tilde{\phi}^{2}}{M}
$$

and $\langle H\rangle=\left(\begin{array}{c}0 \\ \frac{v}{\sqrt{2}}\end{array}\right)$.

\footnotetext{
${ }^{1}$ For other recent discussions on similar frameworks for bi-large mixing, see Refs. $[2,3]$

${ }^{2}$ For some recent discussions of leptogenesis and low energy physics, see Refs. [4]-[8].
} 
The neutrino masses are given by

$$
\mathcal{M}=D^{t r} M_{N}^{-1} D
$$

with

$$
M_{N}=\left(\begin{array}{cc}
M_{1} & 0 \\
0 & M_{2}
\end{array}\right) .
$$

This is exactly the FGY ansatz [1]. The diagonalized neutrino mass matrix is given by

$$
\mathcal{M}^{D}=U_{F G Y}^{t r} \mathcal{M} U_{F G Y}
$$

where

$$
U_{F G Y}=\left(\begin{array}{ccc}
1 / \sqrt{2} & 1 / \sqrt{2} & 0 \\
-1 / 2 & 1 / 2 & 1 / \sqrt{2} \\
1 / 2 & -1 / 2 & 1 / \sqrt{2}
\end{array}\right) \times\left(\begin{array}{ccc}
1 & 0 & 0 \\
0 & \cos \theta & \sin \theta \\
0 & -\sin \theta & \cos \theta
\end{array}\right)
$$

is the FGY neutrino mixing matrix (for the case $b=b^{\prime}$ and $a^{\prime}=\sqrt{2} a$ ). The three neutrino mass eigenvalues are given by

$$
m_{\nu_{3}} \approx 2 b^{2} / M_{2} \gg m_{\nu_{2}} \approx 2 a^{2} / M_{1}, \quad m_{\nu_{1}}=0
$$

and the small angle $\theta \sim m_{\nu_{2}} /\left(\sqrt{2} m_{\nu_{3}}\right)$. Recall, in order to fit neutrino data we have

$$
\begin{aligned}
& m_{\nu_{3}} \approx 0.05 \mathrm{eV}=\sqrt{\Delta m_{\text {atm }}^{2}} \\
& m_{\nu_{2}} \approx 7 \times 10^{-3} \mathrm{eV}=\sqrt{\Delta m_{\text {sol }}^{2}}
\end{aligned}
$$

Although we obtain the FGY ansatz naturally, we cannot explain why $a \sim a^{\prime}$ or $b \sim b^{\prime}$. This difficulty, however, is somewhat reduced in our $S O(10)$ example. In addition, note this model has a straightforward generalization to $S U(5)$ with the conversion of $L_{a}, l_{3} \rightarrow \overline{5}_{a}, \overline{5}_{3}$ and the Higgs doublet $H \rightarrow 5_{H}$. Of course we would then need to discuss charged fermion masses which we will not consider further here. Instead we consider a simple extension to $S O(10)$.

Now consider a similar realization of the same symmetry in an $S O(10)$ SUSY GUT with an $S U(2) \times U(1)$ family symmetry. ${ }^{3}$ The three families of quarks and leptons are contained in three 16 dimensional representations of $S O(10)\left\{16_{a}, 16_{3}\right\}$ with $16_{a}$, $a=$ 1,2 an $S U(2)$ flavor doublet. Consider the charged fermion sector first. Although the charged fermion sector is not the main focus of this letter it is necessary to present a possible superpotential which results in charged fermion masses and mixing angles, since in $S O(10)$ the neutrino and charged fermion sectors are inextricably intertwined.

The superpotential resulting in charged fermion masses and mixing angles is given by

$$
\begin{gathered}
16_{3} 1016_{3}+16_{a} 10 \chi^{a} \\
+\bar{\chi}_{a}\left(M_{\chi} \chi^{a}+45 \frac{\phi^{a}}{\hat{M}} 16_{3}+45 \frac{\tilde{\phi}^{a} \tilde{\tilde{b}}^{b}}{(\hat{M})^{2}} 16_{b}+A^{a b} 16_{b}\right)
\end{gathered}
$$

\footnotetext{
${ }^{3}$ For related charged fermion analyses in $S O(10)$ SUSY GUTS with $S U(2) \times U(1)^{n}$ (or $\left.D_{3}\right)$ family symmetries, see $[9,10]$ (or [11]).
} 
where $M_{\chi}=M_{0}(1+\alpha X+\beta Y)$ includes $S O(10)$ breaking vevs in the $X$ and $Y$ directions, $\phi^{a}, \tilde{\phi}^{a}, A^{a b}=-A^{b a}$ are $S O(10)$ singlet flavon fields with explicit $S U(2)$ properties, $\hat{M}$ is an $S O(10)$ singlet mass and $\langle 45\rangle \sim(B-L) M_{G}$. The fields $\phi, \tilde{\phi}$ are assumed to obtain vevs

$$
\langle\phi\rangle=\left(\begin{array}{c}
\phi^{1} \\
\phi^{2}
\end{array}\right),\langle\tilde{\phi}\rangle=\left(\begin{array}{c}
0 \\
\tilde{\phi}^{2}
\end{array}\right) .
$$

Note, once again, this is completely general, since arbitrary vevs can be rotated into this direction by the $S U(2) \times U(1)$ family symmetry.

The superpotential, (Eqn. 10) results in the following charged fermion Yukawa matrices:

$$
\begin{aligned}
& Y_{u}=\left(\begin{array}{ccc}
0 & \epsilon^{\prime} \rho & -\epsilon \xi \\
-\epsilon^{\prime} \rho & \tilde{\epsilon} \rho & -\epsilon \\
\epsilon \xi & \epsilon & 1
\end{array}\right) \lambda \\
& Y_{d}=\left(\begin{array}{ccc}
0 & \epsilon^{\prime} & -\epsilon \xi \sigma \\
-\epsilon^{\prime} & \tilde{\epsilon} & -\epsilon \sigma \\
\epsilon \xi & \epsilon & 1
\end{array}\right) \lambda \\
& Y_{e}=\left(\begin{array}{ccc}
0 & -\epsilon^{\prime} & 3 \epsilon \xi \\
\epsilon^{\prime} & 3 \tilde{\epsilon} & 3 \epsilon \\
-3 \epsilon \xi \sigma & -3 \epsilon \sigma & 1
\end{array}\right) \lambda
\end{aligned}
$$

with

$$
\begin{aligned}
\xi & =\phi^{1} / \phi^{2} ; \quad \tilde{\epsilon} \propto\left(\tilde{\phi}^{2} / \hat{M}\right)^{2} ; \\
\epsilon & \propto \phi^{2} / \hat{M} ; \quad \epsilon^{\prime} \sim\left(A^{12} / M_{0}\right) ; \\
\sigma & =\frac{1+\alpha}{1-3 \alpha} ; \quad \rho \sim \beta \ll \alpha .
\end{aligned}
$$

It has been shown in Ref. [10] that excellent fits to charged fermion masses and mixing angles are obtained with this Yukawa structure.

In the three $16 \mathrm{~s}$ we have three electroweak doublet neutrinos $\left(\nu_{a}, \nu_{3}\right)$ and three electroweak singlet anti-neutrinos $\left(\bar{\nu}_{a}, \bar{\nu}_{3}\right) .{ }^{4}$ The superpotential $W_{\text {ch.fermions }}$ also results in a neutrino Yukawa matrix:

$$
Y_{\nu}=\left(\begin{array}{ccc}
0 & -\epsilon^{\prime} \omega & \frac{3}{2} \epsilon \xi \omega \\
\epsilon^{\prime} \omega & 3 \tilde{\epsilon} \omega & \frac{3}{2} \epsilon \omega \\
-3 \epsilon \xi \sigma & -3 \epsilon \sigma & 1
\end{array}\right) \lambda
$$

with $\omega=2 \sigma /(2 \sigma-1)$ and a Dirac neutrino mass matrix given by

$$
m_{\nu} \equiv Y_{\nu} \frac{v}{\sqrt{2}} \sin \beta .
$$

\footnotetext{
${ }^{4}$ In an equivalent notation, we have three left-handed neutrinos $\left(\nu_{L a} \equiv \nu_{a}, \nu_{L 3} \equiv \nu_{3}\right)$ and three right-handed neutrinos defined by $\left(\nu_{R a} \equiv \bar{\nu}_{a}^{*}, \nu_{R 3} \equiv \bar{\nu}_{3}^{*}\right)$.
} 
In addition, the anti-neutrinos get GUT scale masses by mixing with three $S O(10) \times$ $S U(2)$ singlets $\left\{N_{i}, i=1,2,3\right\}$. The full superpotential is given by $W=W_{\text {ch.fermions }}+$ $W_{\text {neutrino }}$ with

$$
\begin{gathered}
W_{\text {neutrino }}=\frac{\overline{16}}{\hat{M}}\left(N_{1} \tilde{\phi}^{a} 16_{a}+N_{2} \phi^{a} 16_{a}+N_{3} \theta 16_{3}\right) \\
+\frac{1}{2}\left(S_{1} N_{1}^{2}+S_{2} N_{2}^{2}\right)
\end{gathered}
$$

and we assume $\overline{16}$ obtains a vev $v_{16}$ in the right-handed neutrino direction, $\left\langle S_{i}\right\rangle=M_{i}$ for $i=1,2$ and $\langle\theta\rangle=\theta$. We thus obtain the effective neutrino mass terms given by

$$
W=\nu m_{\nu} \bar{\nu}+\bar{\nu} V N+\frac{1}{2} N M_{N} N
$$

with

$$
V^{t r}=\frac{v_{16}}{\hat{M}}\left(\begin{array}{ccc}
0 & \tilde{\phi}^{2} & 0 \\
\phi^{1} & \phi^{2} & 0 \\
0 & 0 & \theta
\end{array}\right), M_{N}=\operatorname{diag}\left(M_{1}, M_{2}, 0\right)
$$

where $V^{t r}$ is the transpose of $V$. The family symmetry is at least $S U(2) \times U(1)$ where the $S U(2)$ charges are evident, while the $U(1)$ charge assignments for $\left\{\overline{16}, 16_{3}, 16_{a}\right.$, $\left.N_{1}, N_{2}, N_{3}, \phi^{a}, \tilde{\phi}^{a}, \theta, S_{1}, S_{2}\right\}$ are given by $\left\{-2 n_{1}+n_{2}-1,1, x, n_{1}, n_{2}, n_{3}\right.$, $\left.2\left(n_{1}-n_{2}\right)+1-x,\left(n_{1}-n_{2}\right)+1-x, 2 n_{1}-\left(n_{2}+n_{3}\right),-2 n_{1},-2 n_{2}\right\}$.

The electroweak singlet neutrinos $\{\bar{\nu}, N\}$ have large masses of order $V, M_{N} \sim M_{G}$. After integrating out these heavy neutrinos, we obtain the light neutrino mass matrix given by

$$
\mathcal{M}=U_{e}^{t r}\left[m_{\nu}\left(V^{t r}\right)^{-1} M_{N} V^{-1} m_{\nu}^{t r}\right] U_{e} .
$$

It is explicitly defined in the lepton flavor basis where $U_{e}$ is the $3 \times 3$ unitary matrix for left-handed leptons needed to diagonalize $Y_{e}$ (eqn. 12), i.e. $Y_{e}^{D}=U_{e}^{t r} Y_{e} V_{e}^{*}$.

Note,

$$
\left(V^{t r}\right)^{-1}=\frac{\hat{M}}{v_{16}}\left(\begin{array}{ccc}
-1 /\left(\tilde{\phi}^{2} \xi\right) & 1 / \phi^{1} & 0 \\
1 / \tilde{\phi}^{2} & 0 & 0 \\
0 & 0 & 1 / \theta
\end{array}\right)
$$

Let us now define

$$
D^{t r} \equiv m_{\nu}\left(V^{t r}\right)^{-1} M_{N} \mathcal{P}=\left(\begin{array}{cc}
a & 0 \\
a^{\prime} & b \\
0 & b^{\prime}
\end{array}\right)
$$

where

$$
\mathcal{P}=\left(\begin{array}{ll}
1 & 0 \\
0 & 1 \\
0 & 0
\end{array}\right)
$$

and $m_{\nu}$ is the Dirac neutrino mass (Eqn. 15). We then obtain

$$
\mathcal{M}=U_{e}^{t r}\left[D^{t r} \hat{M}_{N}^{-1} D\right] U_{e}
$$


with

$$
\hat{M}_{N} \equiv\left(\begin{array}{cc}
M_{1} & 0 \\
0 & M_{2}
\end{array}\right)
$$

The parameters $\left\{a, a^{\prime}, b, b^{\prime}\right\}$ are given by

$$
\begin{array}{rlrl}
a & \equiv & -\epsilon^{\prime} \omega \lambda\left(M_{1} / \tilde{\phi}^{2}\right) \frac{\hat{M}}{v_{16}} \frac{v \sin \beta}{\sqrt{2}} \\
a^{\prime} & \equiv & \left(-\epsilon^{\prime}\right. & \left.\xi^{-1}+3 \tilde{\epsilon}\right) \omega \lambda\left(M_{1} / \tilde{\phi}^{2}\right) \frac{\hat{M}}{v_{16}} \frac{v \sin \beta}{\sqrt{2}} \\
b & \equiv & \epsilon^{\prime} \omega \lambda\left(M_{2} / \phi^{1}\right) \frac{\hat{M}}{v_{16}} \frac{v \sin \beta}{\sqrt{2}} \\
b^{\prime} & \equiv & -3 \epsilon \xi \sigma \lambda\left(M_{2} / \phi^{1}\right) \frac{\hat{M}}{v_{16}} \frac{v \sin \beta}{\sqrt{2}} .
\end{array}
$$

We have $b \sim b^{\prime}$, since $\epsilon^{\prime} \sim \phi^{1} / \hat{M} \sim \epsilon \xi \sim 3 \tilde{\epsilon} \xi$ (see Eqns. 13 and 28). However, without fine-tuning, we find $a \ll a^{\prime}$. This can be remedied if the implicit order one coefficients in these expressions are used to fine tune the two terms in $a^{\prime}$ so that they cancel to about one part in 10 giving $a^{\prime} \sim a$. Finally, one difference between this $S O(10)$ model and the FGY ansatz is the small mixing angles in $U_{e}$. As a result the observable neutrino mixing matrix $U$ used to diagonalize $\mathcal{M}$ via

$$
\mathcal{M}^{D}=U^{\operatorname{tr}} \mathcal{M} U
$$

is given by

$$
U=U_{e}^{\dagger} U_{F G Y}
$$

where $U_{F G Y}$ is the FGY neutrino mixing matrix (Eqn. 7). Using the results of Blažek et al. (2000) (see Table 2 and discussion in Sect. 4.1) [10] we have

$$
\left|Y_{e}\right| \approx\left(\begin{array}{ccc}
0 & 0.003 & 0.004 \\
0.003 & 0.03 & 0.12 \\
0.004 & 0.12 & 1
\end{array}\right) 0.8
$$

which gives $\left|\left(U_{e}^{t r}\right)_{12}\right| \approx 0.16,\left|\left(U_{e}^{t r}\right)_{23}\right| \approx 0.12$ and $\left|\left(U_{e}^{t r}\right)_{13}\right| \approx 0.01$. Hence, the small mixing angles in $U_{e}$ do not significantly affect the result of bi-large mixing.

However, the main point of FGY is that CP violation, as measured in low energy neutrino oscillation experiments or via the cosmological baryon asymmetry, is governed by the single phase $\delta$ defined by $a^{\prime}=Y a e^{i \delta}$. In the Standard Model or $S U(5)$ examples we retain this nice feature with $\delta=\arg \left(\phi^{2} / \phi^{1}\right)$. In the $S O(10)$ example, although $\delta=\arg \left(\xi^{-1}-3 \tilde{\epsilon} / \epsilon^{\prime}\right)$ with $\xi^{-1}=\phi^{2} / \phi^{1}$, we lose this simple connection since there are generically additional $\mathrm{CP}$ violating angles present in $U_{e}$. Note, however, that now there is the possibility of relating $\mathrm{CP}$ violation in neutrino physics to $\mathrm{CP}$ violation in the $\mathrm{CKM}$ matrix.

In this letter we have presented a "natural" framework for the FGY [1] neutrino mass matrix ansatz. The FGY ansatz has two major virtues,

- it accomodates bi-large neutrino mixing, and 
- it relates $\mathrm{CP}$ violation in neutrino oscillations with the sign of the cosmological baryon asymmetry.

We show that an $S U(2) \times U(1)$ family symmetry can provide the desired FGY neutrino mass ansatz in the MSSM. We have also shown how to obtain an approximate FGY ansatz in an $S O(10)$ SUSY GUT. In this latter example we lose the direct connection between high energy $\mathrm{CP}$ violation made visible by leptogenesis and direct CP violation observable in low energy neutrino oscillations. However since the neutrino sector is now intertwined with the charged fermions, we have other interesting relations. For example, the relation $b \approx b^{\prime}$, needed for a large atmospheric neutrino mixing angle, is now automatic. The large solar mixing angle is, however, still easily accomodated. Moreover, the same $S U(2) \times U(1)$ family symmetry has the virtue of generating the fermion mass hierarchy as well as suppressing large flavor changing neutral current processes $[9,10]$. Finally, it is important to note that our results are not significantly affected by renormalization group $[R G]$ running. It is well known that $R G$ running of the effective dimension 5 lepton-Higgs interaction below the Majorana mass scales $\left[M_{1}, M_{2}\right]$ does not significantly affect the results for neutrino masses and mixing angles when we have a hierarchical neutrino spectrum[12].

\section{Acknowledgements}

Partial support for this work was obtained under DOE grant DOE/ER/01545-840. I greatly benefitted from discussions with P.H. Frampton, J.E. Kim, H.D. Kim, L. Schradin and K. Tobe.

\section{References}

[1] P. H. Frampton, S. L. Glashow and T. Yanagida, Phys. Lett. B 548, 119 (2002) [arXiv:hep-ph/0208157].

[2] R. Kuchimanchi and R. N. Mohapatra, Phys. Rev. D 66, 051301 (2002) [arXiv:hepph/0207110].

[3] S. F. King, arXiv:hep-ph/0211228.

[4] J. R. Ellis and M. Raidal, Nucl. Phys. B 643, 229 (2002) [arXiv:hep-ph/0206174].

[5] T. Endoh, S. Kaneko, S. K. Kang, T. Morozumi and M. Tanimoto, Phys. Rev. Lett. 89, 231601 (2002) [arXiv:hep-ph/0209020].

[6] W. Buchmuller, P. Di Bari and M. Plumacher, Phys. Lett. B 547, 128 (2002) [arXiv:hep-ph/0209301].

[7] M. Raidal and A. Strumia, Phys. Lett. B 553, 72 (2003) [arXiv:hep-ph/0210021].

[8] G. C. Branco, R. Gonzalez Felipe, F. R. Joaquim, I. Masina, M. N. Rebelo and C. A. Savoy, arXiv:hep-ph/0211001. 
[9] R. Barbieri, L. J. Hall, S. Raby and A. Romanino, Nucl. Phys. B 493, 3 (1997) [arXiv:hep-ph/9610449].

[10] T. Blazek, S. Raby and K. Tobe, Phys. Rev. D 60, 113001 (1999) [arXiv:hepph/9903340]; Phys. Rev. D 62, 055001 (2000) [arXiv:hep-ph/9912482].

[11] R. Dermisek and S. Raby, Phys. Rev. D 62, 015007 (2000) [arXiv:hep-ph/9911275].

[12] P. H. Chankowski and S. Pokorski, Int. J. Mod. Phys. A 17, 575 (2002) [arXiv:hep$\mathrm{ph} / 0110249]$ and references therein. 